\title{
Olhando além do campo: 0 desenvolvimento da agenda de pesquisa da midiatização
}

\section{Looking beyond the field: development of the mediatization research agenda}

\author{
Entrevista com STIG HJARVARD* \\ University of Copenhagen, Department of Media, Cognition and Communication, Copenhague, Dinamarca \\ por NICOLÁS L LANO LINARES** \\ Universidade de São Paulo, Programa de Pós-Graduação em Comunicação, São Paulo-SP, Brasil
}

1

O LONGO DA última década, Stig Hjarvard tem sido um dos principais defensores da perspectiva institucional na teoria da midiatização. Seu trabalho a respeito do referencial teórico e das manifestações empíricas da dinâmica dos processos de midiatização em diferentes campos ampliou o debate sobre a relação entre evolução da mídia e as transformações de instituições culturais e sociais no contexto da modernidade. É co-presidente no Departamento de Mídia, Cognição e Comunicação na Universidade de Copenhague. É coordenador da seção da ECREA sobre midiatização. Tem escrito e organizado vários livros, incluindo A midiatização da cultura e da sociedade e Mediatization and religion (co-organizado com Mia Lövheim, Nordicom, 2012). Debatemos com o professor Hjarvard o potencial e os equívocos da teoria da midiatização, o desenvolvimento de estudos da mídia e da comunicação na região nórdica e as vantagens de encontros interdisciplinares.

MATRIZes: Na América Latina, o primeiro programa de pós-graduação em estudos da comunicação surgiu em 1972 (Universidade de São Paulo); seu currículo inicial foi definido principalmente pelas ligações interdisciplinares entre os estudos/campos de literatura e artes. Do ponto de vista institucional, o principal objetivo era legitimar o estudo da comunicação e de textos-cultura populares no ambiente acadêmico. $\mathrm{O}$ senhor poderia traçar o histórico dos estudos da mídia e contar como foi a construção e a legitimação do campo

\footnotetext{
* Professor no Departamento de Mídia, Cognição e Comunicação da Universidade de Copenhague, Copenhague, Dinamarca. Escreveu, entre outros livros, A midiatização da cultura e da sociedade (Porto Alegre: Unisinos, 2014). E-mail: stig@hum. ku.dk

** Doutorando em ciências da comunicação na Universidade de São Paulo. Seus interesses de pesquisa incluem cultura alimentar e material, discursos de mídia e jornalísticos e explorações críticas da cultura visual. É um dos editores de Antropologia \& Comunicação (2014). É membro ativo do grupo de pesquisa GESC3: estudos semióticos em comunicação, cultura e consumo. E-mail: nllano@usp.br

English revision: Lucas Pinto
}

MATRIZes 
acadêmico em mídia e comunicação na Dinamarca e, de modo mais amplo, na região nórdica?

Stig Hjarvard: Como parece ter acontecido na América Latina e no Brasil, parte dos estudos de mídia e comunicação surgiu das ciências humanas, especialmente dos estudos literários, que se concentrou em expandir a noção de objetos textuais e culturais em seu sentido mais amplo. Algumas pessoas vinham dos estudos literários, outras da história; eu venho da literatura comparada. Além disso, alguns saíram da linguística e outros campos relacionados. Essa foi uma das incursões das humanidades, principalmente dos estudos literários.

Outra importante incursão foi um grupo menor, dentro dos estudos de comunicação política, que estava interessado na forma como a mídia intervinha nos processos eleitorais, olhando para o papel da comunicação de massa; a televisão, o jornal e outros meios, questionando seus critérios. Esses estudiosos exerceram alguma influência, sobretudo nas escolas de jornalismo. Era outro ramo, não tão grande, mas ainda assim reconhecível no campo.

Por fim, houve uma terceira incursão, vinda dos estudos de marketing/ negócios, em que pessoas do segmento publicitário - e também de várias formas de escolas de administração - estavam interessadas em como analisar a segmentação de público e as preferências dos consumidores, se as peças estavam sendo direcionadas às pessoas certas, e assim por diante. Acho que essas foram as três incursões, e cada uma ainda tem uma base em diversas universidades e escolas de negócios.

Claramente, as ciências humanas foram a incursão mais proeminente nos estudos de mídia e comunicação, não apenas na Dinamarca, mas também na Noruega e, em certa medida, na Suécia. Na Suécia, dá-se maior ênfase à abordagem das ciências sociais aos estudos de mídia e comunicação, especialmente na comunicação política. A Noruega e a Dinamarca têm uma forte base nas humanidades, assim como nos estudos literários. Mas, com o passar dos anos as coisas mudam, e surge um diálogo entre os diversos grupos. É interessante ver que, dentro das humanidades, as pessoas que se ocupavam de questões relacionadas a mídia e comunicação eram muitas vezes vistas por outros, das ciências humanas também, como os sociólogos. Afinal, estavam interessadas no impacto profundo da mídia e da comunicação na sociedade e na cultura. Deve-se ter em mente que, de fora, elas de fato pareciam vir das ciências humanas. Sempre houve essa tensão sobre a verdadeira origem dos estudos de mídia e comunicação. Seria nas humanidades? Nas ciências sociais?

Ultimamente, tem havido uma incursão também da tecnologia. A completa datificação da comunicação com ideias da ciência da computação e da informação propiciou uma nova incursão, com discussões interessantes sobre 
mídia e comunicação em termos de TI e tecnologia. Acho que esse é um quarto desenvolvimento, mas claramente posterior aos outros.

MATRIZes: Sua formação acadêmica é em estudos literários e de mídia. O senhor tem trabalhado com diversos objetos/temas, como jornalismo, história da mídia, globalização e mídia (culturas midiáticas globais), e na última década foi um dos principais proponentes da teoria de midiatização, especialmente sua tradição institucional. Seus estudos nunca se centraram em representações midiáticas (apesar de ter trabalhado com discurso jornalístico e institucional); sempre houve ênfase nos padrões mutáveis das relações sociais, com base em pesquisas empíricas, principalmente entre instituições (mídia, jornalismo), sociedade e cultura. Como e por que o senhor passou a dar maior atenção ao desenvolvimento teórico da midiatização?

Hjarvard: Em diversos trabalhos anteriores, estive muito preocupado com questões empíricas, incluindo representações midiáticas. Esses estudos concentravam-se na mídia dinamarquesa e escandinava - especialmente na mídia jornalística -, mas trabalhos empíricos não costumam circular tão bem na comunidade internacional de pesquisas quanto contribuições teóricas. Sempre busquei combinar análise empírica com considerações teóricas, de várias maneiras. Acho que uma questão que sempre me instigou foi que, de certa forma, precisávamos de um campo mais amplo para compreender a influência da mídia e da comunicação. Muitos trabalhos de estudos de mídia e comunicação centraram-se em representações, mas precisamos considerar também os aspectos estruturais e históricos.

Acho que a teoria dos estudos de mídia e comunicações se desenvolveu de uma forma em que, do meu ponto de vista, buscava cada vez mais respostas em si mesma, porque foi imensamente bem-sucedida enquanto disciplina autônoma. Deve estar entre os três mais bem-sucedidos casos de institucionalização de campo científico das últimas décadas, se não $o$ maior. Em um brevíssimo espaço de tempo, diversas universidades adotaram programas de mídia e comunicações, que cresceram e se tornaram muito influentes, também porque vários estudantes estão fazendo cursos de mídia e comunicação. Nesse processo, o campo, por razões óbvias, desenvolveu seu próprio currículo, horizonte e estrutura - é isso que acontece quando se institucionaliza um campo de pesquisa como um programa educacional. Como consequência, o campo passou a se concentrar um tanto demasiadamente sobre o próprio mundo da mídia e da comunicação, em vez de tentar compreender como mídia e comunicação influenciam e interagem com o mundo exterior.

Acho que a proposta da midiatização é exatamente fornecer, se não uma resposta, pelo menos uma pauta para discutir a influência entre mídia e desen- 
volvimentos de comunicação, de um lado, e desenvolvimento cultural e social, do outro. Talvez meu interesse nessa perspectiva se deva em parte também ao fato de eu vir de fora. Minha formação é diferente da situação atual, em que todos os estudantes que recebemos aqui e os jovens pesquisadores que empregamos são produto de nosso próprio campo de investigação. Eles saem com um conhecimento em mídia e comunicação. Há muitos benefícios nisso. Não vou argumentar contra esse fato. Mas um dos problemas pode ser o risco de a pessoa, de alguma forma, ter uma ideia um pouco mais restrita sobre o que são mídia e comunicação, considerando que, quando se vem de fora, pode-se ter um quadro mais amplo para compreender sua importância, e ao mesmo tempo reconhecer os limites dos estudos desse tema.

MATRIZes: Eis uma boa introdução para minha primeira pergunta sobre midiatização. Qual é o potencial da teoria da midiatização? Livingstone e Lunt (2015) e o senhor (2014) argumentaram que poderia ser uma contribuição significativa para as ciências sociais. Qual é sua opinião sobre o que a teoria da midiatização e, mais amplamente, os estudos de mídia e comunicação podem oferecer ao campo mais abrangente das ciências sociais e humanas?

Hjarvard: Na minha opinião, a midiatização como suporte teórico pode oferecer um quadro macrossociológico para se investigar, de várias maneiras, como a mídia, a cultura e a sociedade interagem. A teoria sugere proposições gerais sobre como ocorre essa interação. Ao mesmo tempo, acho que a verdadeira investigação de como ocorre a midiatização e como ela se dá no seio da família e das comunicações políticas tem de ser feito no que Merton (1968) rotula de nível de médio alcance.

Não podemos esperar resultados gerais dos processos de midiatização porque há muitas variáveis em jogo. Inúmeras condições contextuais influenciam o uso e a institucionalização reais desses processos. Precisamos estar muito mais embasados no contexto e no empírico para realizar as pesquisas. No entanto, precisamos de um quadro geral para discutir esses desenvolvimentos e fazer uma comparação, entre os diversos campos, de como as coisas são explicitadas.

Essa é a força da midiatização como conceito. Da mesma forma, assim como outros conceitos semelhantes - como globalização, individualização etc. -, deve fornecer uma perspectiva macrossocial sobre algumas tendências gerais. A ideia de globalização ou midiatização como conceitos não é fornecer um espaço teórico fechado ou ser uma teoria confinada em si mesma. $\mathrm{Na}$ verdade, são necessários vários outros conceitos teóricos, como, por exemplo, a teoria da esfera pública ou a teoria das redes. Muitos trabalhos feitos tanto 
dentro como fora dos estudos de mídia e comunicação são bastante úteis para nossa compreensão dos processos de midiatização.

A ideia não é que devemos começar tudo de novo, como se nosso conhecimento acumulado e os conceitos existentes não importassem. A midiatização como estrutura é muito mais uma tentativa intelectual de analisar criticamente e sintetizar os resultados de pesquisas já realizadas, assim como de nos permitir rearticular algumas questões fundamentais que os estudos de mídia e comunicação não foram suficientemente capazes de responder.

Há uma enormidade de coisas boas por aí, então meu argumento não é que sem midiatização não conseguiremos compreender nada. É mais no sentido de que ela pode nos ajudar a entender por que mídia e comunicação assumiram um lugar mais proeminente nas sociedades contemporâneas em comparação a tempos atrás. Também é um conceito que espero que seja útil para outras disciplinas, a fim de compreender o que está acontecendo em seus campos. Talvez paradoxalmente, acho que as pessoas fora das escolas de mídia e comunicação ou desse campo acadêmico estão, em certo sentido, mais receptivas à noção de midiatização do que as de dentro. Parece-me que esse conceito serve como uma ponte para outras disciplinas, a fim de esclarecer por que mídia e comunicação podem ser mais importantes agora do que antes.

MATRIZes: Como o senhor disse, a estrutura teórica da midiatização não é uma tabula rasa para os problemas de estudos em mídia e comunicação que foram discutidos na última metade do século passado. De certa forma, uma das tarefas dos acadêmicos trabalhando sob a égide da midiatização é reformular pesquisas e trabalhos acadêmicos anteriores. Isso é algo que o senhor vê como uma tarefa importante nesse estágio do desenvolvimento da teoria?

Hjarvard: Sim. É um dos grandes desafios agora: tentar fortalecer e qualificar o argumento levando em consideração alguns estudos realizados fora do quadro da midiatização. Isso já foi feito, no sentido de que o que eu e alguns acadêmicos temos defendido também se baseia em pesquisas realizadas por estudiosos de mídia e de outras disciplinas. Considero muito frutíferos esses estudos que tentaram combinar uma abordagem histórica com uma abordagem sociológica. Um exemplo muito proeminente é o estudo de Jürgen Habermas da Mudança estrutural da esfera pública (1989), em que tenta combinar argumentos históricos e sociológicos. Há algumas mudanças históricas ocorrendo por um longo período em que a constelação entre vários domínios institucionais é alterada. Essa combinação de história e sociologia é fecunda para o projeto da midiatização. 
MATRIZes: O senhor colocaria John B. Thompson (1995) também nessa categoria?

Hjarvard: Sim, claro. Não acho que seja coincidência ele também ter essa ideia. Ele chama de midiazação, mas aponta para o mesmo processo, de que a mídia tornou-se proeminente em determinada fase da modernidade. Ele enfatiza a midiatização como parte integrante da modernidade, incluindo uma maior diferenciação da sociedade, e isso torna mídia e comunicação importantes para a coordenação interna de vários setores, mas também entre setores da sociedade num plano mais amplo. Acho muito importante considerar a midiatização dentro do contexto da modernidade, porque, caso contrário, faríamos vista grossa a algumas das principais dinâmicas a que está relacionada a midiatização. Esta é uma das linhas divisórias que existem atualmente entre as pessoas que gostariam de fazer uma análise muito mais prolongada, civilizacional da midiatização, olhando-a a partir do despertar da civilização, e aquelas que amarram a questão da midiatização a processos da modernidade. Tal distinção claramente tem consequências para o argumento histórico.

MATRIZes: Considerando a diferença entre essa tradição que pensa a midiatização de uma perspectiva maior, histórica, e o senhor, que trabalha com a dimensão institucional, gostaria de ouvir sua opinião sobre a influência da mídia e o efeito produzido por outros macro/metaprocessos de alta modernidade. Em um artigo de 2008 (The mediatization of society: a theory of the media as agents of social and cultural change), o senhor falou sobre midiatização direta e indireta. Ainda pensa todo o processo considerando esses dois graus distintos de efeito? Na minha opinião, o senhor parece ter desenvolvido uma compreensão mais complexa de todo o processo.

Hjarvard: Espero que todos tenhamos desenvolvido argumentos. A distinção entre midiatização direta e indireta foi baseada em observações empíricas de que a midiatização deve abarcar várias maneiras sobre como a interação social pode ser influenciada pela mídia. Portanto, a distinção original foi apresentada para dizer que existem áreas em que há uma midiatização mais direta ocorrendo, porque as pessoas agora usam a mídia para determinadas práticas que não usavam antes. Essas práticas agora têm de ser realizadas pelo uso da mídia. E existem outras práticas sociais em que a mídia não está no centro da atividade. No entanto, as práticas são pelo menos condicionadas culturalmente de uma forma ou de outra pelo ambiente da mídia. Mas talvez todo o desenvolvimento geral da atualidade, a propagação veloz de várias formas de mídia móvel e interativa - smartphones, redes sociais e outras -, a informatização de nossas vidas, em certo sentido tornou essa distinção menos relevante. 
Fica cada vez mais difícil pensar em qualquer tipo de interação social que não envolva, de alguma forma, o uso de mídia. Portanto, a distinção é dificultada porque muitas mais áreas da vida são diretamente mediadas de uma maneira ou de outra e, portanto, sujeitas à influência das lógicas dos diversos meios de comunicação. Não estou dizendo que a distinção não pode ser útil. Pode continuar a sê-lo para fins analíticos. Talvez, em um nível global, não mais seja a distinção mais importante, porque a propagação da mídia interativa e móvel envolveu a mídia em ainda mais formas de interação social.

MATRIZes: Há um monte de comentários críticos sobre seu trabalho a respeito das lógicas midiáticas. É um dos conceitos sobre os quais mais se debateu nos últimos anos. A recente conferência sobre as lógicas midiáticas, realizada na Universidade de Bonn, em que o senhor foi um dos palestrantes convidados - ao lado de David L. Altheide e Friedrich Krotz -, sugere que essas lógicas midiáticas ainda detêm um valor conceitual para compreender o processo geral de midiatização da cultura e da sociedade bem como a dinâmica específica encontrada em diferentes domínios de análise. O senhor poderia, por favor, compartilhar sua perspectiva sobre o desenvolvimento do conceito e sua importância para a definição do processo de midiatização de contextos e domínios institucionais?

Hjarvard: Acho que a noção de lógicas midiáticas é útil porque focaliza nossa atenção nas várias formas em que a mídia trabalha e influencia outras áreas da vida. Talvez algumas críticas à noção de lógicas midiáticas se devam a um mau entendimento do termo. Como já disse algumas vezes, é importante entendê-lo como uma espécie de metáfora. Não devemos lê-lo literalmente, e acho que várias formas de práticas de mídia - por exemplo, o jornalismo funcionam de maneiras completamente similares às leis da lógica formal ou do raciocínio matemático.

Uma leitura literal das lógicas midiáticas seria inadequada. $\mathrm{Na}$ minha opinião, é mais uma metáfora que usamos para pensar a mídia por meio de alguns procedimentos identificáveis, de natureza formal e informal. Regras formais são dadas pela regulação da mídia, por políticas, pelas leis de mercado etc. Mas a mídia também é regulada por várias regras informais - como o conhecimento prático, as culturas organizacionais, as convenções profissionais de jornalistas, convenções estéticas da dramaturgia, entre outras - que informam a maneira como as pessoas trabalhando com mídia fazem as coisas. Além disso, há também alguns aspectos tecnológicos dessa lógica.

Da forma como vejo - e isso é o que tenho defendido atualmente -, se quisermos olhar para as lógicas midiáticas através de um vocabulário mais ge- 
ral, poderíamos traduzi-lo no que a sociologia rotula de regras e recursos. Poderíamos entender a lógica midiática como as regras e recursos por meio dos quais a mídia funciona. Isso envolve dimensões institucionais, leis, estruturas de poder etc. Envolve dimensões estéticas. Envolve aspectos tecnológicos materiais: há certas coisas que se pode fazer com uma tecnologia, e há certas coisas que não se pode fazer. A constelação de regras e recursos institucionais, estéticas e tecnológicas torna-se a lógica midiática.

Nesse sentido, acho que lógicas midiáticas é um termo proveitoso. Ajuda-nos a entender como outros domínios da sociedade são influenciados por sua acomodação à lógica da mídia e também como a mídia, de certa forma, acomoda-se à lógica de outras esferas sociais. Não é que eu esteja apaixonado pela noção de lógica midiática; prefiro problematizar que, sem uma noção que especifique as regras e recursos por meio dos quais a mídia trabalha, seria difícil explicar sua importância no âmbito da política, da religião e de outros domínios da sociedade. Se não perguntarmos como funcionam as várias tecnologias e instituições de mídia, como podemos entender sua importância para a cultura e a sociedade?

MATRIZes: Se um dos pressupostos da teoria da midiatização é que a mídia tem um papel nas transformações históricas nos âmbitos social e cultural, um quadro teórico das ciências sociais - juntamente com dados empíricos - deve ser usado para compreender essas transformações. Na minha opinião, essa é uma perspectiva que o senhor compartilha com o trabalho de Nick Couldry (2014). O senhor concorda com esse posicionamento?

Hjarvard: Por exemplo, Nick Couldry e alguns outros argumentam que seria mais útil o que eles rotulam de perspectiva prática. No entanto, ainda não sei de caso algum que tenha se baseado em teoria da prática que nos permita gerar conhecimento sobre como mídia e cultura em geral, dentro de determinado contexto, possam interagir uma com a outra. O problema da teoria da prática é que ela acaba enfatizando demasiadamente uma abordagem de baixo para cima de nosso entendimento de questões sociais e culturais e, portanto, dá pouca atenção às dimensões estruturais e coletivas dos processos de midiatização. Nunca chega ao ponto em que se pode dizer algo de natureza mais geral. Como afirmado anteriormente, prefiro o nível de alcance médio de pesquisas empíricas para construir uma teoria, pois isso nos permite generalizar em um nível que é tanto ambicioso em termos de teoria como é fundamentado no empírico.

Para mim, também aponta para a força do conceito de lógica midiática. Se não for lido literalmente, mas pensado como uma metáfora para guiar a análise das regras e recursos por meio dos quais a mídia trabalha, tem-se o 
nível adequado de generalização. Não se estende demasiadamente na teoria nem ignora totalmente o empírico.

MATRIZes: O senhor estava falando sobre a compreensão falha do conceito de lógica midiática. Uma crítica recente ao conceito de midiatização por Deacon e Stanyer $(2014 ; 2015)$ mostra que, longe de ser um conceito solidificado e incontestado, a midiatização ainda está tentando impor sua pauta no campo da mídia e comunicação (e, em menor grau, nas ciências sociais). De certa forma, isso trouxe de volta algo que o senhor comentou sobre o entendimento falho dos principais conceitos de uma teoria geral da midiatização. Ainda temos de lidar com essa compreensão falha do conceito? E mais, falta autocrítica na teoria da midiatização em relação a seus próprios argumentos?

Hjarvard: Como um dos defensores da perspectiva da midiatização, deixo aos outros o papel de juiz sobre a falta de autocrítica. Desde seu surgimento, é claro, um conceito como midiatização deveria e deve evocar críticas. Lembro-me da discussão, 25 anos atrás, sobre globalização, na qual houve um debate feroz, intenso entre pessoas discutindo o termo, se era útil ou não, suas implicações normativas etc. Por meio desse debate, temos uma compreensão muito mais matizada e fecunda do que significa globalização em um nível conceitual e do que pode ela implicar em diferentes partes do mundo.

A conclusão geral foi que a globalização é útil como uma espécie de estrutura geral para abordar um conjunto de questões importantes. Não se pode falar de globalização como um processo unificado e singular. É preciso contextualizá-la caso se deseje analisar o que está acontecendo, tanto em termos geográficos como em termos do domínio em que se está.

Vejo as discussões atuais sobre midiatização de forma semelhante; estamos em meio a um debate frutífero que irá reforçar e qualificar nossa noção de midiatização. Penso, no entanto, que algumas críticas foram inadequadas: Deacon e Stanyer afirmam, com razão, que a noção de midiatização é muitas vezes usada de forma um tanto vaga ou imprecisa por alguns. Tenho de concordar com isso, mas de certa forma não se pode desconsiderar um conceito por ser usado por algumas pessoas de modo bem superficial. Se for feita uma análise semelhante de periódicos acadêmicos usando os termos individualização ou globalização, serão encontrados muitos casos em que os pesquisadores não definiram adequadamente individualização ou globalização. Não deixo de considerar tais conceitos com base nisso, mas esse foi, na verdade, o principal argumento de Deacon e Stanyer.

Nesse sentido, não a considero uma intervenção produtiva, mas claramente pesquisas sobre midiatização também devem ser verificadas. Na minha opinião, os críticos deram enorme ênfase à noção de midiatização como um 
desenvolvimento impressionante e arrebatador da mídia, que chega a colonizar todos os aspectos do mundo. Logicamente é preciso investigar essa questão, mas acredito que os críticos interpretaram erroneamente a ideia de midiatização. Vendo pelo lado positivo, parece-me que, quanto mais as pessoas leem textos sobre o tema, menos atenção dão a esses argumentos deturpados. Então se inicia uma discussão muito mais interessante, sobre como poderíamos realmente entender a relação entre a evolução da mídia e as transformações das instituições culturais e sociais.

MATRIZes: O senhor coorganizou recentemente um livro chamado The dynamics of mediatization conflicts (2015). É interessante analisar conflitos através das lentes da midiatização, porque nos permite compreender diferentes graus e dinâmicas da influência da mídia com base em contextos geográficos distintos. $\mathrm{O}$ senhor poderia falar um pouco sobre a coletânea de artigos publicados nesse livro?

Hjarvard: Certamente não foi nossa invenção falar sobre conflito midiatizado. Simon Cottle é um dos principais estudiosos da questão. Nosso objetivo foi fornecer uma coletânea de estudos que tentam combinar duas vertentes de pesquisa: estudos de midiatização com uma tradição conhecida e já bem estabelecida de estudar mídia e conflitos.

Um foco importante do livro é olhar para a maneira como as chamadas novas mídias alteraram as condições para o surgimento de conflitos. Ao longo dos anos, foram publicados diversos estudos sobre a maneira como os meios de comunicação de massa influenciaram conflitos, em termos de estrutura, de definição de pauta etc.

Acho que agora temos uma situação nova, com a presença de várias formas de mídia móvel e interativa que permite que muitos mais atores se envolvam em um conflito. Essa mudança geral nas condições para um conflito é, a meu ver, um fenômeno da midiatização para ser estudado separadamente.

No entanto, também é importante salientar que, quando alguém começa a estudar a maneira como determinados conflitos ocorrem ou se iniciam, ele está predominantemente estudando processos de mediação, e não de midiatização. Está examinando como as pessoas realmente se comunicam umas com as outras, ou não se comunicam, ou se comunicam mal - e isso é parte importante dessa análise. Todavia, acho importante que o estudo da midiatização se coloque em posição em que possa ser útil para o estudo real dos processos de mediação.

Nesse sentido, a midiatização também serve para condicionar a maneira como interagimos uns com os outros, e conflitos são interessantes porque ten- 
dem a engrossar e destacar determinados problemas e relações de poder que estão mudando ou sendo criados. Se a pessoa, por exemplo, quiser comparar conflitos atuais com exemplos anteriores na era dos meios de comunicação de massa, há estudos interessantes baseados na teoria das redes e na noção de ações conectivas, sugerida por Bennett e Segerberg (2012), muito fecundos para compreender essas novas condições de conflitos. São bons exemplos de estudiosos contribuindo para pesquisas de midiatização sem usar esse termo. Para mim, é exatamente isso que os novos meios de comunicação móveis e digitais alteraram: eles condicionam a maneira como conflitos se desenrolam; e, em particular, o que é conhecido como ação conectiva, em comparação à ação coletiva, é um fenômeno novo.

É importante observar que a midiatização pode implicar uma nova condição, mas ela não determina a forma como as ações são realizadas. Acho que essa é uma distinção importante a se fazer; pelo que entendo, em termos de conflitos, a midiatização pode propiciar novas condições para o desenrolar dos conflitos, mas não determina como eles devem ser desenrolados. O resultado de conflitos pode ser muito diferente entre um contexto e outro, portanto não é possível prever alguns resultados devido às novas situações; eles estão se condicionando de maneira diferente. Por exemplo, permitir que mais atores se envolvam - mas também com o potencial de controlar os fluxos de vários meios de comunicação em rede - é um fator importante para definir como se pode realmente influenciar e controlar o desenvolvimento de conflitos.

Só para dar um exemplo, durante a famosa ou infame crise da charge de Maomé aqui na Dinamarca - isso já faz dez anos -, uma das lições aprendidas pelo Ministério de Relações Exteriores dinamarquês foi a necessidade de um novo tipo de diplomacia. Até então, tinha se apoiado nas formas tradicionais, em que nações conversavam entre si, com canais oficiais da diplomacia, mas nesse caso entendeu-se claramente que algo novo estava acontecendo. Era muito menos controlável e passível de contenção pelos meios de comunicação tradicionais, então foi necessário introduzir o que rotularam de diplomacia pública, por meio da qual poderiam aproveitar uma variedade de mídias, tanto as de massa como as redes sociais, para tentar influenciar vários atores durante esses tipos de crise. Acho que essa é a maneira como a mídia consegue condicionar o desenrolar de conflitos, mas o resultado desses conflitos não pode ser determinado pelo processo de midiatização.

MATRIZes: Esse livro surgiu no grupo Midiatização da Cultura, certo? Hjarvard: Sim. 
MATRIZes: O grupo tem atuado faz cinco anos. Seus projetos têm continuado agora que acabou o financiamento?

Hjarvard: Tínhamos uma área de pesquisa prioritária dentro do departamento, do qual eu era chefe, e então surgiu essa bolsa do Conselho de Pesquisa dinamarquês, que reúne pesquisadores de três universidades - Copenhague, Aarhus e Aalborg - e dura cinco anos. Não vamos renová-la da forma como está, mas com certeza vamos prosseguir com a discussão sobre midiatização. Um sucesso importante no ano passado foi termos institucionalizado a midiatização como um campo de pesquisa, tanto no contexto dinamarquês como no europeu.

Acho que uma consequência muito clara disso foi que a Associação Europeia de Educação e Pesquisa em Comunicação (ECREA) tornou-a uma seção permanente. Isso garante que teremos um espaço para discutir tais questões, e, assim, não precisaremos tentar desenvolver pequenos fóruns nos mais variados lugares para manter o debate ativo. Agora foi institucionalizado e amplamente reconhecido como um tópico distinto. Portanto, estou bastante otimista quanto ao futuro da discussão, porque agora chegamos a um momento em que ela pode se desenvolver.

MATRIZes: Há algum projeto em vista? Sei de um call for papers para um novo livro sobre teoria da midiatização que será publicado no próximo ano (Dynamics of mediatization: understanding cultural and social change). Em que mais o senhor está trabalhando?

Hjarvard: Ah, sim. Existem alguns outros projetos. Atualmente estou envolvido em um projeto comparativo de conflitos midiatizados relacionados à religião na Suécia, na Dinamarca e na Noruega. Vai me ocupar pelos próximos três anos. Situa-se principalmente na Noruega, mas estou liderando a parte dinamarquesa da pesquisa, e há um grupo na Suécia também. Este é um projeto em que estou envolvido, e há a seção da ECREA e suas atividades. Atualmente, estou pensando em colocar mais ênfase no futuro na forma como a mídia, entendida como meio de comunicação em rede, vai criar novas condições para nós nos relacionarmos social e culturalmente com o outro. Gostaria de combinar a perspectiva da midiatização com a teoria das redes.

Por exemplo, há vários estudiosos holandeses, como Jan Van Dijck (2012) e José Van Dijck e seu trabalho sobre culturas de conectividade (2013), com argumentos que podem ser bem aproveitados na análise da midiatização.

Uma maneira de fazer isso seria admitir que estamos cada vez mais vivenciando - tomando de empréstimo um conceito de Habermas - uma mídia que busca a integração entre o que ele chama de sistema e o mundo da vida. Se 
pensarmos em termos sociológicos, os sistemas como atividades organizadas, institucionalizadas, que trabalham sob uma forma específica de lógica burocrática, comercial, política etc., conclui-se que existem vários tipos de normas de comunicação real. Cada vez mais, acho que a mídia de rede propicia novas formas de unir esses dois aspectos.

Por exemplo, quando se pensa em mídia de rede social, há uma variedade de comunicações e interações bastante mundanas, ordinárias e humanas, atividades diárias da vida real, que cada vez mais também são sustentadas por estruturas da mídia, não só pelas tais normas de vida real, mas também por diversas formas de lógicas comerciais, tecnológicas e outras. Numa rede social, o ato de tornar-se amigo de alguém não só é direcionado por uma iniciativa humana, espontânea, da vida real de fazer amigos, mas certamente também é realizado por várias formas de algoritmos e imperativos comerciais de se fazer amigos.

Considero essa uma das maneiras interessantes por meio das quais a midiatização pode fornecer novas ideias sobre o modo como as diversas mídias de rede - a que estamos cada vez mais incorporados - mudam a forma como interagimos uns com os outros, não só na política, em termos de instituições religiosas, mas também em um nível bastante interpessoal. $\mathbf{M}$

\section{REFERÊNCIAS}

BENNETT, W. L.; SEGERBERG, A. The logic of connective action. Information, Communication \& Society, v. 15, n. 5, p. 739-768, 2012. DOI: http:// dx.doi.org/10.1080/1369118X.2012.670661

COULDRY, N. Mediatization and the future of field theory. In: LUNDBY, K. (org.). Mediatization of Communication. Berlim/Boston: De Gruyter. Mouton, 2014. p. 227-245.

DEACON, D.; STANYER, J. Mediatization: key concept or conceptual bandwagon. Media, Culture \& Society, v. 36, n. 7, p. 1032-1044, 2014. DOI: http://dx.doi.org/10.1177/0163443714542218

DEACON, D.; STANYER, J. 2015. "Mediatization and" or "Mediatization of"? A response to Hepp et al. Media, Culture \& Society, v. 37, n. 3, p. 655-657, 2015. DOI: http://dx.doi.org/10.1177/0163443715580761

ESKJÆR, M., F; HJARVARD, S.; MORTENSEN, M. (orgs.). The dynamics of mediatized conflicts. Nova York: Peter Lang, 2015.

HABERMAS, J. The structural transformation of the public sphere. Cambridge: Polity Press, 1989. (Publicado originalmente em alemão em 1962).

HJARVARD, S. The mediatization of society: a theory of the media as agents of social and cultural change. Nordicom Review, v. 29, n. 2, p. 105-134, 2008. 
Disponível em: $<$ http://www.nordicom.gu.se/sites/default/files/kapitel-pdf/269_hjarvard.pdf>. Acesso em: 3 mar. 2016.

HJARVARD, S. Mediatization and cultural and social change: an institutional perspective. In: LUNDBY, K. (org.). Mediatization of communication. Berlim/Boston: De Gruyter. Mouton, 2014, p. 199-226.

LUNT, P.; LIVINGSTONE, S. Is "mediatization" the new paradigm for our field? A commentary on Deacon and Stanyer $(2014,2015)$ and Hepp, Harvard and Lundby. Media, Culture and Society, 2015. No prelo. Disponível em: <http://eprints.lse.ac.uk/63409/>. Acesso em: 3 mar. 2016.

MERTON, R. K. Social theory and social structure. Enlarged edition. Nova York: The Free Press, 1968.

THOMPSON, J. B. The media and modernity, a social theory of the media. Cambridge: Polity, 1995.

VAN DIJCK, José. The culture of connectivity: a critical history of social media. Oxford: Oxford University Press, 2013.

VAN DIJCK, Jan. The network society. Londres: SAGE, 2012. 